\title{
Partial polarization switching in ferroelectrics-semiconductors with charged defects
}

\author{
A.N. Morozovska, Eu.A. Eliseev ${ }^{*}$, E. Cattan ${ }^{* *}$, D. Remiens**, \\ V. Lashkaryov Institute of Semiconductor Physics, NAS of Ukraine, \\ 41, pr. Nauky, 03028 Kyiv, Ukraine \\ E-mail:_morozo@mail.i.com.ua \\ ${ }^{*}$ Institute for Problems of Materials Science, National Academy of Sciences of Ukraine, \\ 3, Krjijanovskogo str., 03142 Kyiv, Ukraine, \\ ${ }^{* *} I E M N$, UMR 8520 OAE-dept/ MIMM, Universite de Valenciennes et du Hainaut-Cambresis, \\ Le Mont Houy, 59313 Valenciennes Cedex 9, France
}

\begin{abstract}
We propose the phenomenological description of ferroelectric disordering caused by charged defects in ferroelectric-semiconductors. The good agreement between the obtained experimental results for PZT films and theoretical calculations has been shown.

We suppose that proportional to the averaged charge density $\bar{\rho}_{S}$ of defects improper conductivity is sufficiently high to provide the screening of charge density random fluctuations $\delta \rho_{s}$ in the absence of external field. When external electric field $E_{0} t^{\prime}$ is applied, inner field fluctuations and induction fluctuations $\delta D$ appear in the inhomogeneously polarized system "charged fluctuation + screening cloud".

We show that the macroscopic state of ferroelectric-semiconductor with random charged defects and sufficiently high improper conductivity can be described by three coupled equations for three order parameters. Averaged over sample volume induction $\bar{D}$ determines the ferroelectric ordering in the system, its square fluctuation $\overline{\delta D^{2}}$ determines disordering caused by electric field fluctuations appeared around charged fluctuations $\delta \rho_{s}$, and $\overline{\delta D \delta \rho_{s}}$ reflects the correlations between the free carriers screening cloud and charged defects $\delta \rho_{s}$. For the first time, we derive the following system of three coupled equations:

$\Gamma \frac{\partial \bar{D}}{\partial t}+\left(\alpha+3 \beta \overline{\delta D^{2}}\right) \bar{D}+\beta \bar{D}^{3}=E_{0}(t), \frac{\Gamma}{2} \frac{\partial}{\partial t} \overline{\delta D^{2}}+\left(\alpha+3 \beta \bar{D}^{2}\right) \overline{\delta D^{2}}+\beta\left(\overline{\delta D^{2}}\right)^{2}=-E_{0}(t) \frac{\overline{\left(\delta \rho_{s} \delta D\right)}}{\bar{\rho}_{s}}$ $\Gamma \frac{\partial}{\partial t} \overline{\delta D \delta \rho_{s}}+\left(\alpha+3 \beta \bar{D}^{2}+\beta \overline{\delta D^{2}}\right) \overline{\delta D \delta \rho_{s}}=-E_{0}(t) \frac{\overline{\delta \rho_{s}^{2}}}{\bar{\rho}_{s}}$.

Also the obtained system of coupled equations qualitatively describes the peculiarities of polarization switching (footprint and minor hysteresis loops) in such ferroelectric materials with charged defects as PZT films with growth imperfections, PLZT ceramics and SBN single crystals doped with cerium.
\end{abstract}

Keywords: ferroelectrics-semiconductors, charged defects, partial switching, footprint and minor hysteresis loop.

Paper received 24.04.04; accepted for publication 21.10.04.

\section{Introduction}

In most cases, stable partial switching of the spontaneous polarization can be achieved in imperfect ferroelectric materials [1]. Sometimes, the polarization reversal process is strongly asymmetric even in thick ferroelectric films and bulk samples, and in particular the minor hysteresis loops appear in imperfect ferroelectrics (see e.g. [2-5]). For the doped ferroelectrics, this phenomena is strongly dependent on the type and concentration of dopants [3] and the external field frequency [2,3]. For the system of sandwich type metal-ferroelectrics semiconductor [5], this type of switching is defined by the depolarization field and built-in charge layer in the ferroelectrics - semiconductor interface [6]. The similar system was theoretically studied in [7] allowing for the semiconductor properties of ferroelectrics. The model of 


\section{A.N. Morozovska et al.: Partial polarization switching in ferroelectrics-semiconductors ...}

ref. [7] predicts that only one direction of spontaneous polarization is stable with the ferroelectric layer thickness decrease.

Another interesting feature of the polarization switching in ferroelectrics with non-isovalent dopants are the clamped or pinched hysteresis loops observed in La-doped lead zirconate titanate sol-gel films after annealing them in hydrogen atmosphere [8]. This type of hysteresis loops are sometimes called as footprint loops, their appearance was attributed to the influence of the sample conductivity [9]. Constricted hysteresis loops similar to those observed by authors of ref. [8] were found in the La-doped lead zirconate titanate ceramics with composition near the morphotropic boundaries between ferroelectric tetragonal, rhombohedral and antiferroelectric phases [10]. This effect was attributed to the structural changes from cubic matrix with embedded microdomains to orthorhombic macrodomain state twice during one switching cycle of the external field. However, we do not know the adequate quantitative model describing the hysteresis loops with constrictions.

We would like to underline that in all the aforementioned materials where footprint and minor loops exist either non-isovalent impurities or some unavoidable imperfections manifest themselves as charged defects. Moreover, these "dusty" materials would be rather considered as improper semiconductor than ideal dielectrics with random electric fields [11]. These two facts give the basis of our model.

We propose the new phenomenological model that can give both the simple qualitative explanation and analytical description of partial polarization switching phenomena in bulk ferroelectric materials with charged defects and sufficiently high improper conductivity. We try to involve the minimum number of hypothesis into our model. Moreover, we have not used the detailed description of the chemical nature, concentration and sizes distribution of randomly situated immovable charged defects that are the sources of movable charge carriers, inner electric field and induction fluctuations. Also our model admits continuous transformation from the ordered ferroelectric to the disordered material under increasing the charged defects concentration fluctuations.

The main goal of our paper is to demonstrate that macroscopic state of the thick sample with random charged defects can be described by the system of three coupled equations, which is similar to such well-known nonlinear systems of first order differential equations as the Lorenz one [12]. Such dynamical systems of equations can reveal chaotic regimes, strange attractors as well as strongly non-ergodic behaviour and continuous relaxation time spectrum. Up to date, we have studied only the stability of the system stationary states by means of the reduced free energy and simulated quasi-equilibrium ferroelectric hysteresis loops. The good agreement between the theoretical calculations and obtained experimental results for thick PZT films has been shown. Certainly, the dynamical dielectric response of this first obtained system requires further investigations.

\section{The problem}

We assume that unavoidable charged defects or nonisovalent impurity atoms are embedded into hypothetical "pure" uniaxial ferroelectric. We suppose also that even in the absence of proper conductivity, imperfections provide a rather high improper conductivity in the bulk of the sample. The concentration of these atoms $\rho_{s}$ fluctuates due to the great variety of misfit effects (different ionic radii, local symmetry breaking, clusterization). These fluctuations $\delta \rho_{s}$ are considered in the continuous medium framework, i.e., the discrete charge density (point charges in Scheme 1) is approximated by the smooth function $\rho_{s}=\bar{\rho}_{s}+\delta \rho_{s}$. In this approach, the short-range fluctuations will be neglected, and the smallest period $d$ in $\delta \rho_{s}$ spatial spectrum is much greater than the average distance $h$ between real point defect atoms.

Hereinafter, we regard embedded defects almost immovable and charged with density $\rho_{s}(\mathbf{r})$. The sample as a whole is electro-neutral. In this case, the movable free charges $n(\mathbf{r}, t)$ surround each charged impurity center (see Scheme 1). The characteristic size of these screening clouds is of the same order as the Debye screening radius $R_{D}$. For the large enough average defect concentration $\bar{\rho}_{s}$, radius $R_{D} \sim 1 / \sqrt{\bar{\rho}_{s}}$ is much smaller than the average distance $h$ between defects. It is obvious that in the absence of the external electric field $E_{0}$ the inner field is close to zero outside the screening clouds (see Scheme $1 a, c)$. But when one applies the external field $E_{0}$, the screening clouds of free charges are deformed, and nanosystem "defect center + screening cloud" becomes polarized (Scheme $1 b, d)$. If the defect charge density fluctuations $\delta \rho_{s}(\mathbf{r})$ are absent, the short-range electric fields caused by homogeneously distributed induced dipoles are canceled, and no long-range electric field arises in the bulk of the sample (Scheme $1 b$ ). Moreover, the fluctuations $\delta \rho_{s}(\mathbf{r})$ do not reveal themselves in the absence of the external filed $E_{0}$ due to the complete screening by movable space-charge fluctuations $\delta n$ (Scheme $1 \mathrm{c}$ ). Contrary to this at $E_{0} \neq 0$ polarized regions " $\delta \rho_{s}(\mathbf{r})+\delta n(\mathbf{r}, t)$ " cause the long-range inner electric field fluctuations $\delta E(\mathbf{r}, t)$ (Scheme $1 d)$. According to the equations of state, the fluctuations of the inner electric field $\delta E$ cause induction fluctuations $\delta D$. The rigorous conditions that determine the existence of these induction fluctuations will be given below.

Evidently non-homogeneous mechanical stresses appearing near the defects should be taken into account [13]. But the consideration of non-homogeneous mechanical stresses significantly complicates the problem, and we hope that the system behavior will not change qualitatively under the influence of non-homogeneous mechanical stresses. It is also known (see e.g. [14]) that homogeneous elastic stresses due to the electrostriction coupling with the polar order parameter can be taken into account by the renormalization of the free energy expansion coefficients. 


\section{A.N. Morozovska et al.: Partial polarization switching in ferroelectrics-semiconductors ...}

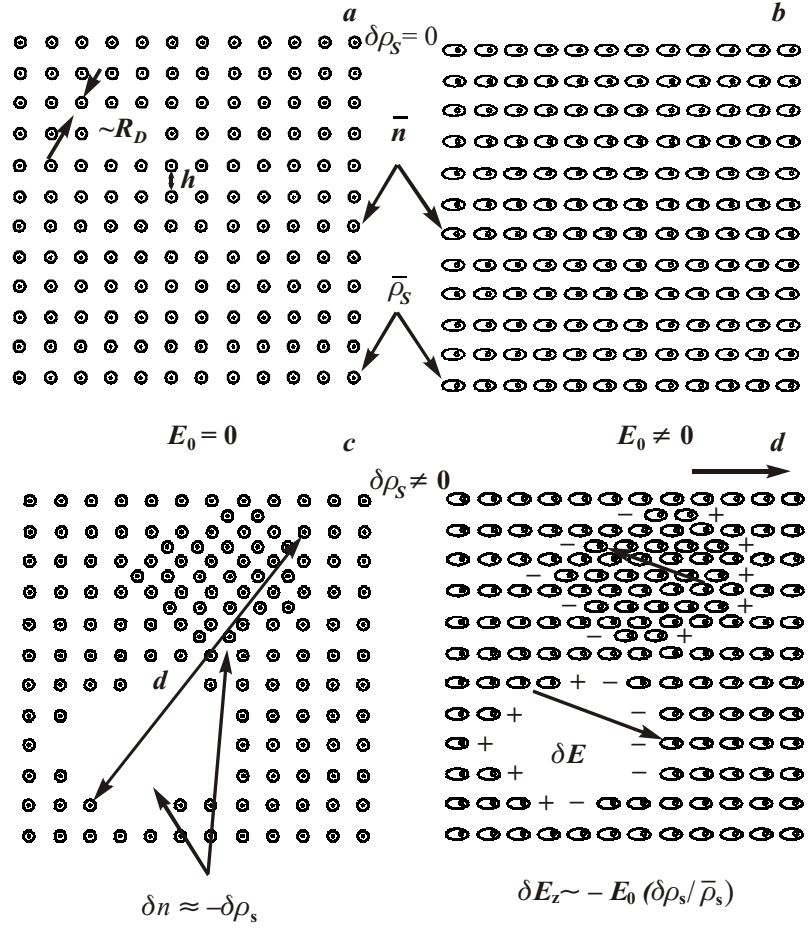

Scheme 1. The system of charged defects with the charge density $\rho_{S}$ (dots) screened by the free charges with density $n$ (circles or ellipses) and screening radius $R_{D}$. Parts $\boldsymbol{a}, \boldsymbol{b}$ represent charges homogeneous distributions $\left(\delta \rho_{s}=0, \delta n=0\right)$. Parts $\boldsymbol{c}, \boldsymbol{d}$ represent the distribution with the long-range fluctuations $\left(\delta \rho_{s} \neq 0, \delta n \neq 0\right)$ and the space period $d$ much greater than the average distance $h$ between defects. The parts $\boldsymbol{a}, \boldsymbol{c}$ and $\boldsymbol{b}, \boldsymbol{d}$ show the system with the zero and nonzero external field $E_{0}$, respectively.

\section{General equations}

Maxwell's equations for the electric induction $\mathbf{D}$, field $\mathbf{E}$ and equation of continuity have the form:

$\operatorname{div} \mathbf{D}=4 \pi \rho, \operatorname{rot} \mathbf{E}=0, \frac{\partial \rho}{\partial t}+\operatorname{div} \mathbf{j}_{c}=0$. state:

They have to be supplemented by the equations of

$\mathbf{D}_{\perp}=\varepsilon_{\perp} \mathbf{E}_{\perp}, \quad D_{z}=D_{S}+\varepsilon_{z}(D) E_{z}$,

$\mathbf{j}_{c}=\sum_{m}\left(\mu_{m} \rho_{m} \mathbf{E}-\kappa_{m} \operatorname{grad} \rho_{m}\right), \quad \rho=\sum_{m} \rho_{m}+\rho_{s}$.

The sample is regarded as linear dielectric in transverse $x, y$-directions and as nonlinear polar material in longitudinal $z$-direction. Here $\varepsilon_{\perp}$ is transversal component of dielectric permittivity, $\rho_{m}, \mu_{m}$ and $\kappa_{m}$ are the $m$ type movable charge volume density $(m=n, p)$, mobility and diffusion coefficient, respectively, $\mathbf{j}_{c}$ is the macroscopic free-carriers current, $\rho_{s}(\mathbf{r})$ is the given charge density of static defects.

The spatial-temporal distribution of the induction zcomponent $D_{z} \equiv D$ can be obtained from the LandauGinsburg-Khalatnikov equation:
$\Gamma \frac{\partial D}{\partial t}+\alpha D+\beta D^{3}-\gamma \frac{\partial^{2} D}{\partial \mathbf{r}^{2}}=E_{z}$.

Here $\Gamma>0$ is the kinetic coefficient, $\alpha(T)=\alpha_{T}(T-$ $\left.-T^{*}\right), T$ is the absolute temperature, $T^{*}$ is the Curie temperature of the hypothetical pure (free of defects) sample, $b>0, g>0$. Equations (1), (2) can be rewritten as:

$\frac{\partial}{\partial z} D=4 \pi \rho-\varepsilon_{\perp} d i v \mathbf{E}_{\perp}$,

$\operatorname{div}\left[\sum_{m=e, h}\left(\mu_{m} \rho_{m} \mathbf{E}-\kappa_{m} \operatorname{grad} \rho_{m}\right)+\frac{1}{4 \pi} \frac{\partial}{\partial t}\left(D \mathbf{e}_{z}+\varepsilon_{\perp} \mathbf{E}_{\perp}\right)\right]=0$.

Here $\mathbf{e}_{z}$ is the unit vector directed along $z$-axis.

Hereinafter, we suppose that homogeneous external field $E_{0}(t)$ is applied along polar $z$-axis. The sample occupies the region $-\ell<z<\ell$, i.e., it is infinite in the transverse directions. Let us consider that the electrodes potential difference $\varphi=2 \ell E_{0}(t)$ is independent on transverse coordinates. So, the inner field satisfies the conditions:

$$
\frac{1}{2 \ell} \int_{-\ell}^{\ell} E_{z}(\mathbf{r}, t) d z=E_{0}(t), \quad \int \mathbf{E}_{\perp}(\mathbf{r}, t) d \mathbf{r}_{\perp}=0 .
$$

Boundary conditions depend on the mechanism of the spontaneous induction screening, which associates with the formation of oppositely charged space-charge layers with thickness $\ell_{c}$ [15]. We can assume that the induction distribution is symmetrical for a rather thick sample $\left(\ell>>\ell_{c}\right)$ with equivalent boundaries $z= \pm \ell$, i.e.:

$$
\frac{1}{S} \int_{S} D\left(\mathbf{r}_{\perp}, z=\ell, t\right) d \mathbf{r}_{\perp} \approx \frac{1}{S} \int_{S} D\left(\mathbf{r}_{\perp}, z=-\ell, t\right) d \mathbf{r}_{\perp} .
$$

$S$ is the sample cross-section. We also introduce the averaging over the sample volume:

$f(\mathbf{r}, t)=\overline{f(t)}+\delta f(\mathbf{r}, t), \quad \bar{f}(t)=\frac{1}{V} \int_{V} f(\mathbf{r}, t) d \mathbf{r}$.

Hereinafter, the dash designates the averaging over the sample volume $V, f=\left\{\rho, \rho_{m}, \rho_{s}, E, D, j, \ldots\right\}, \overline{\delta f(\mathbf{r}, t)}=0$. All the functions $\delta f(\mathbf{r}, t)$ consist of the regular part caused by spontaneous induction screening [15] and the random one caused by fluctuations. Since the contribution from the screening region $|z|>\ell-\ell_{c}$ to the integrals $\int_{V} \delta f^{n}(\mathbf{r}, t) d \mathbf{r}$ is negligibly small for the rather thick sample $\ell \gg>\ell_{c}$, and $\delta f$ is the fast oscillating function in the remainder of the sample $|z| \leq \ell-\ell_{c}$, one can conclude that:

$$
\overline{\delta f^{2 n}(\mathbf{r}, t)} \sim\left(\overline{\delta f^{2}(\mathbf{r}, t)}\right)^{n}, \quad n=1 ; 2 \ldots
$$

$$
\overline{\delta f^{2 n+1}(\mathbf{r}, t)} \approx 0, \quad n=1 ; 2 \ldots
$$


Also, we suppose that the correlation between the different $\delta f$-functions is equal to zero, if the total power of the functions is an odd number.

It follows from (5) and (4) that:

$E_{z}(\mathbf{r}, t)=E_{0}(t)+\delta E_{z}(\mathbf{r}, t)$,

$\mathbf{E}_{\perp}(\mathbf{r}, t)=\delta \mathbf{E}_{\perp}(\mathbf{r}, t)$

i.e., $\bar{E}$ is the applied uniform field $E_{0}(t)$ and $\overline{\mathbf{E}}_{\perp}=0$. Notice that the average values $E, D$ are determined experimentally $[15,16]$ in most cases. Having substituted (6)-(8) into (5) and averaged, one can obtain the expressions for the average quantities, namely:

$$
\begin{aligned}
& \bar{\rho}=0 \Rightarrow \sum_{m=e, h} \bar{\rho}_{m}=-\bar{\rho}_{s}, \\
& \overline{\mathbf{j}}(t)=\overline{\mathbf{j}}_{c}(t)+\mathbf{e}_{z} \frac{\partial}{\partial t} \frac{\bar{D}(t)}{4 \pi} .
\end{aligned}
$$

The absence of the space charge average density $\bar{\rho}$ follows from the sample electro-neutrality and corresponds to the result [15], [17]. Here $\overline{\mathbf{j}}(t)$ is the total macroscopic current. Using (5)-(7) one can obtain:

$$
\begin{aligned}
& \frac{\partial}{\partial z} \delta D=4 \pi\left(\sum_{m=e, h} \delta \rho_{m}+\delta \rho_{s}(\mathbf{r})\right)-\varepsilon_{\perp} \operatorname{div} \delta \mathbf{E}_{\perp} \\
& \sum_{m=e, h}\left(\mu_{m}\left[\delta \rho_{m} E_{0} \mathbf{e}_{z}+\left(\bar{\rho}_{m}+\delta \rho_{m}\right) \delta \mathbf{E}-\overline{\delta \rho_{m} \delta \mathbf{E}}\right]-\kappa_{m} \operatorname{grad} \delta \rho_{m}\right)+ \\
& +\frac{1}{4 \pi} \frac{\partial}{\partial t}\left(\mathbf{e}_{z} \delta D+\varepsilon_{\perp} \delta \mathbf{E}_{\perp}\right)=0 .
\end{aligned}
$$

Using the nonlinear equation (4) and formulae (8)(11), it is easy to obtain the following system of equations:

$$
\begin{aligned}
& \Gamma \frac{\partial \bar{D}}{\partial t}+\left(\alpha+3 \beta \overline{\delta D^{2}}\right) \bar{D}+\beta \bar{D}^{3}=E_{0}(t), \\
& \Gamma \frac{\partial}{\partial t} \delta D+\left(\alpha+3 \beta \bar{D}^{2}\right) \delta D+3 \beta \bar{D}\left(\delta D^{2}-\overline{\delta D^{2}}\right)+ \\
& +\beta \delta D^{3}-\gamma \frac{\partial^{2} \delta D}{\partial \mathbf{r}^{2}}=\delta E_{z} .
\end{aligned}
$$

The system of equations (13)-(16) is complete, because the quantities $\delta \rho_{m}, \delta \mathbf{E}$ can be expressed via the fluctuations of induction $\delta D$ and $\delta \rho_{s}(\mathbf{r})$ allowing for (13), (14). It determines the spatial-temporal evolution of the induction in the bulk sample and has to be supplemented by the initial distributions of all variables.

The system (13)-(16) can be used to study the mechanisms of domain wall pinning by the given distribution of charged defects fluctuations $\delta \rho_{s}(\mathbf{r})$, domain nucleation during spontaneous induction reversal in the ferroelectric semiconductors with non-isovalent impurities. These problems for the ferroelectric ideal insulators were considered earlier in detail (see e.g. [18], [19]). Hereinafter, we consider only the average characteristics of the system.

\section{Coupled equations}

In order to simplify the nonlinear system (13)-(16), the following hypotheses have been used.

a) The sample is the improper semiconductor with rather high $n$-type conductivity:

$$
\begin{aligned}
& \sum_{m=e, h} \rho_{m} \approx-\bar{\rho}_{s}+\delta n, \quad \bar{n} \approx-\bar{\rho}_{s}, \\
& \overline{\delta n}=0, \quad \mu<0, \quad \bar{\rho}_{s}>0 .
\end{aligned}
$$

Hereinafter, we neglect the proper conductivity and omit the subscript " $m$ ".

b) The equations (13)-(14) can be linearized with respect to $\delta n$ in the bulk of the sample, where $\delta E \sim \delta n$ and so:

$|\delta n \delta \mathbf{E}-\overline{\delta n \delta \mathbf{E}}|<<\bar{\rho}_{s} \cdot|\delta \mathbf{E}|$.

c) The characteristic time of $\delta n, \delta \mathbf{E}, \delta D$ and $E_{0}$ changing is the same order as the maxwellian time, which is much smaller than the Landau-Khalatnikov relaxation time:

$\frac{1}{4 \pi \mu \bar{\rho}_{s}}<<\frac{\Gamma}{|\alpha|}$.

d) We suppose also that the smallest period $d$ of the inhomogeneities distribution (see Scheme 1) is much greater than the Debye screening radius $R_{D}=$ $=\sqrt{-\kappa / 4 \pi \varepsilon_{\perp} \mu \bar{\rho}_{s}}$ and correlation length (the thickness of the neutral domain wall) $\ell_{c}=\sqrt{\gamma / \alpha}$, namely

$$
R_{D}^{2} / d^{2}<<1, \ell_{c}^{2} / d^{2}<<1 .
$$

Note that for the typical defects concentration $\sim 1-10 \%$ that provides sufficiently high improper conductivity at the room temperature $R_{D} \sim 5 \mathrm{~nm}$ [15], [17], $d \sim 50 \mathrm{~nm}$, $\ell_{c} \sim 1 \mathrm{~nm}$ [13], [15], i.e. inequalities (20) is valid.

After neglecting the temporal derivatives of $\delta \mathbf{E}$ and $\delta D$ (compare with [17]), linearization over $\delta n$ and elementary transformations, the equations (13)-(14) acquire the form:

$$
\begin{aligned}
& \delta \mathbf{E} \approx \mathbf{e}_{z} E_{0}(t) \frac{\delta n}{\bar{\rho}_{s}}-\frac{\kappa}{\mu \bar{\rho}_{s}} \operatorname{grad}(\delta n), \\
& \delta n-\varepsilon_{\perp} \frac{\kappa}{4 \pi \mu \bar{\rho}_{s}} \Delta_{\perp} \delta n \approx \frac{1}{4 \pi} \frac{\partial}{\partial z} \delta D-\delta \rho_{s} .
\end{aligned}
$$

The gradient terms in (21b) can be neglected in accordance with (20), because $R_{D}^{2}=-\kappa /\left(4 \pi \varepsilon_{\perp} \mu \bar{\rho}_{S}\right)$ is transverse Debye screening radius (see Scheme 1). Moreover, if only the concentration of free carriers is high enough to provide the good screening of the charged inhomogeneities $\delta \rho_{s}$, the gradient of the induction fluctuations is small in the bulk of a sample (see Scheme 2), $(1 / 4 \pi) \partial \delta D / \partial z \cong\left(\delta \rho_{s}+\delta n\right)$ namely:

$$
\left|\frac{1}{4 \pi} \frac{\partial}{\partial z} \delta D\right|<<\left|\delta \rho_{s}\right|
$$




\section{A.N. Morozovska et al.: Partial polarization switching in ferroelectrics-semiconductors ...}

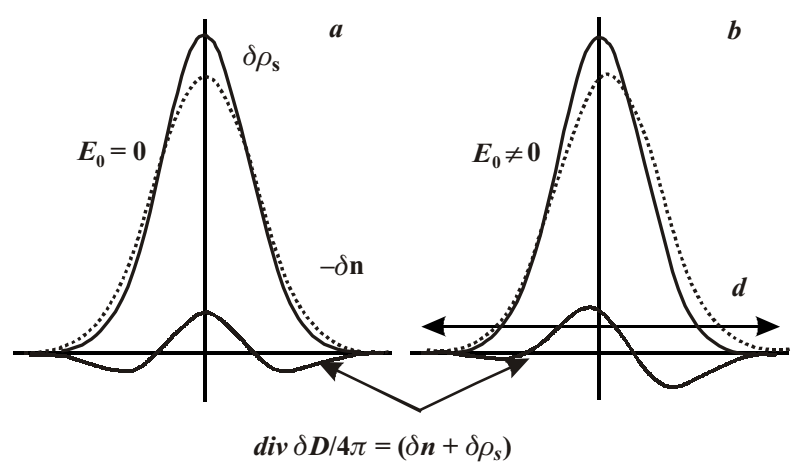

Scheme 2. The screening of the charged defects $\delta \rho_{s}$ by free charges $\delta n$.

Thus, the field variation $\delta E_{z}$ from (21a) can be expressed via $\delta D$ and $\delta \rho_{s}$ (see Appendix A and [20]):

$\delta E_{z} \approx \frac{\kappa}{\mu} \frac{\partial}{\partial z}\left(\frac{\delta \rho_{s}}{\bar{\rho}_{s}}\right)-E_{0}(t) \frac{\delta \rho_{s}}{\bar{\rho}_{s}}$.

Having substituted solution (23) into (16), we obtain from (15)-(16) the self-consistent system of the nonlinear integral-differential equations for $D$ and $\delta D$. Its nonhomogeneity is proportional to charge fluctuations $\delta \rho_{s}$ and external field $E_{0}$.

The approximate system of first-order differential equations for average induction $\bar{D}$, its square fluctuation $\delta D^{2}$ and correlation $\overline{\delta D \delta \rho_{s}}$ can be derived after some elementary transformations (see Appendix A). Thus, we obtain three coupled equations:

$\Gamma \frac{\partial \bar{D}}{\partial t}+\left(\alpha+3 \beta \overline{\delta D^{2}}\right) \bar{D}+\beta \bar{D}^{3}=E_{0}(t)$,

$$
\begin{aligned}
& \frac{\Gamma}{2} \frac{\partial}{\partial t} \overline{\delta D^{2}}+\left(\alpha+3 \beta \bar{D}^{2}\right) \overline{\delta D^{2}}+ \\
& +\beta\left(\overline{\delta D^{2}}\right)^{2}=-E_{0}(t) \frac{\overline{\left(\delta \rho_{s} \delta D\right)}}{\bar{\rho}_{s}} \\
& \Gamma \frac{\partial}{\partial t} \overline{\delta D \delta \rho_{s}}+\left(\alpha+3 \beta \bar{D}^{2}+\beta \overline{\delta D^{2}}\right) \times \\
& \times \overline{\delta D \delta \rho_{s}}=-E_{0}(t) \frac{\overline{\delta \rho_{s}^{2}}}{\bar{\rho}_{s}}
\end{aligned}
$$

The system (24) determines the temporal evolution of the bulk sample dielectric response and have to be supplemented by the initial values of $\bar{D}, \overline{\delta D^{2}}$ and $\overline{\delta D \delta \rho_{s}}$ at $t=0$.

Coupled equations (24) have the following physical interpretation (compare with modified approach [20]). The macroscopic state of the bulk sample with charged defects can be described by three parameters: $\bar{D}, \overline{\delta D^{2}}$ and $\overline{\delta D \delta \rho_{s}}$. The long-range order parameter $\bar{D}$ describes the ferroelectric ordering in the system, and the dis- order parameter $\overline{\delta D^{2}}$ describes disordering caused by inner electric fields arising near charged non-homogeneities $\delta \rho_{s}$. The correlation $\overline{\delta D \delta \rho_{s}}$ determines the correlations between the movable screening cloud $\delta n$ and static charged defects $\delta \rho_{s}$.

We will show that equations (24) admit the continuous transformation from the perfect ferroelectric $\left(\delta \rho_{s} \rightarrow 0\right.$ and so $\left.\overline{\delta D^{2}}(t) \rightarrow 0\right)$ to the local disordering $\left(\overline{\delta \rho_{s}^{2}} \neq 0\right.$ and so $\left.\overline{\delta D^{2}}(t) \neq 0\right)$ and then to the completely disordered material $\left(\overline{\delta D^{2}}(t)>|\alpha| / 3 \beta\right)$ under the increase of fluctuations $\delta \rho_{s}$.

As a resume to this section, we would like to stress that the derived non-Hamiltonian system of coupled equations (24) is similar to other well-known nonlinear systems of first order differential equations (e.g., the Lorenz system). Such dynamical systems possess chaotic regions, strange attractors as well as strongly non-ergodic behaviour and continuous relaxation time spectrum [12]. Any new system of such type demands a separate detailed mathematical study that was not the aim of this paper. Hereinafter, we discuss only the system behaviour in the vicinity of the equilibrium states far from the possible chaotic regions.

\section{Quasi-stationary states}

Let us consider the stationary solution of (24), which corresponds to changing the quasi-static external field. It is easy to check that the system (24) is not the Hamiltonian one, i.e., it can not be directly obtained by varying of some free energy functional. But in order to study the stability of the stationary points under the external field changing, we try to obtain the reduced free energy.

Let us exclude one of the order parameters from the system (24). Indeed we can easy obtain from (24c) that

$$
\overline{\delta D \delta \rho_{s}}=-\frac{E_{0} \cdot \overline{\delta \rho_{s}^{2}}}{\bar{\rho}_{s}\left(\alpha+3 \beta \bar{D}^{2}(t)+\beta \overline{\delta D^{2}}\right)} .
$$

Substituting (25) into (24b) and extracting square root one obtains

$$
\sqrt{\overline{\delta D^{2}}} \cdot\left(\alpha+3 \beta \overline{D^{2}+\beta \overline{\delta D^{2}}}\right)= \pm E_{0} \sqrt{\overline{\delta \rho_{s}^{2}} / \bar{\rho}_{s}^{2}} .
$$

In the stationary case (24a), it acquires the form:

$$
\left(\alpha+3 \beta \overline{\delta D^{2}}\right) \bar{D}+\beta \bar{D}^{3}=E_{0} .
$$

It is easy to see that system of equations (25b), (25c) can be obtained from the variational principle. Really, the equations (25b), (25c) can be integrated over averaged induction fluctuations $\sqrt{\overline{\delta D^{2}}}$ and induction $\bar{D}$, and so the reduced free energy functional determining the stability of the stationary points has the following form: 


$$
\begin{aligned}
& G\left(\bar{D}, \sqrt{\overline{\delta D^{2}}}\right)=\frac{\alpha}{2}\left(\bar{D}^{2}+\overline{\delta D^{2}}\right)+\frac{\beta}{4}\left(\bar{D}^{4}+\left(\overline{\delta D^{2}}\right)^{2}\right)+ \\
& +\frac{3 \beta}{2} \bar{D}^{2} \cdot \overline{\delta D^{2}}-E_{0}\left(\bar{D}+\sqrt{\frac{\overline{\delta \rho_{s}^{2}} \cdot \overline{\delta D^{2}}}{\bar{\rho}_{s}^{2}}}\right) .
\end{aligned}
$$

Hereinafter, we suppose that the root $\sqrt{\overline{\delta D^{2}}}$ can be both negative and positive depending on the external conditions. It is easy to verify that the equations of state

$$
\frac{\partial}{\partial \bar{D}} G\left(\bar{D}, \sqrt{\overline{\delta D^{2}}}\right)=0, \frac{\partial}{\partial \sqrt{\overline{\delta D^{2}}}} G\left(\bar{D}, \sqrt{\overline{\delta D^{2}}}\right)=0
$$

coincide with the equations (25c) and (25b). Let us rewrite energy functional (26a) in dimensionless variables.

$G_{m}\left(D_{m}, \Delta_{D}\right)=-\frac{1}{2}\left(D_{m}^{2}+\Delta_{D}^{2}\right)+\frac{1}{4}\left(D_{m}^{4}+\Delta_{D}^{4}\right)+$

$+\frac{3}{2} D_{m}^{2} \cdot \Delta_{D}^{2}-E_{m}\left(D_{m}+R \cdot \Delta_{D}\right)$.

Here $\alpha<0, D_{m}=\bar{D} / D_{S}, D_{S}=\sqrt{-\alpha / \beta}, E_{m}=E_{0} /\left(-\alpha D_{S}\right)$, $\Delta_{D}=\sqrt{\delta D^{2}} / D_{S}, R=\sqrt{\overline{\delta \rho_{s}^{2}} / \bar{\rho}_{s}^{2}}$.

The contour plots of negative values of the free energy (27) at different $E_{m}$ amplitudes and small $R$ value are depicted in Figs 1, 2.

The free energy minimums (crosses and stars) prove the existence of two sorts of stable states: the first one with non-zero averaged induction values $\bar{D} \neq 0$ and
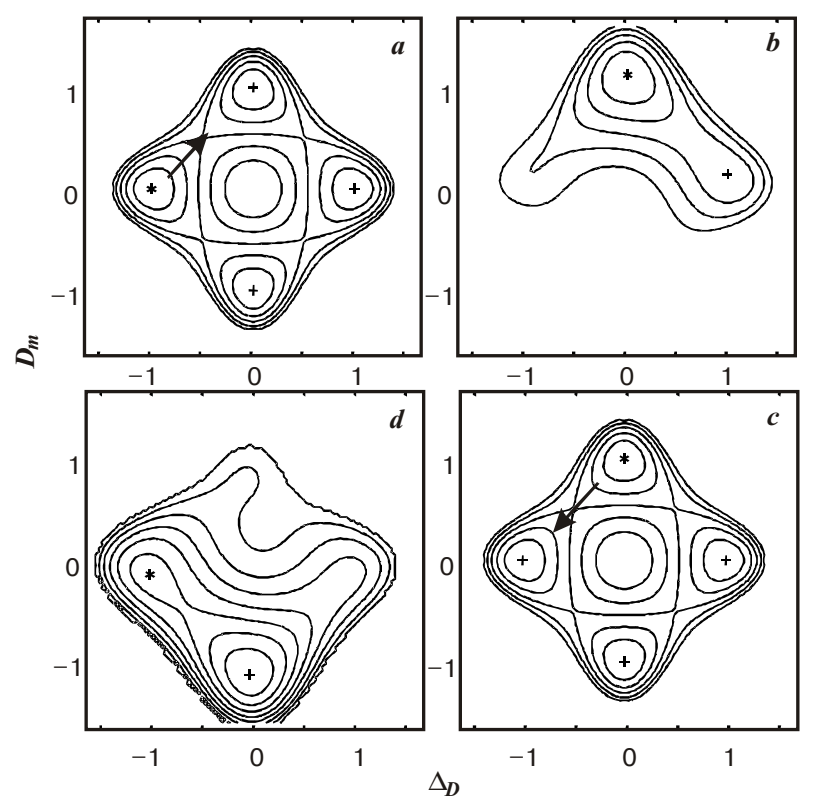

Fig. 1. The contour plots of negative values of the free energy (27) at $R^{2}=0.1$ and different values of $E_{m}=0,0.3,0,-0.3$ (parts $a, b, c, d$, respectively). Crosses denote the positions of minima; asterisk is the position of the current minimum.
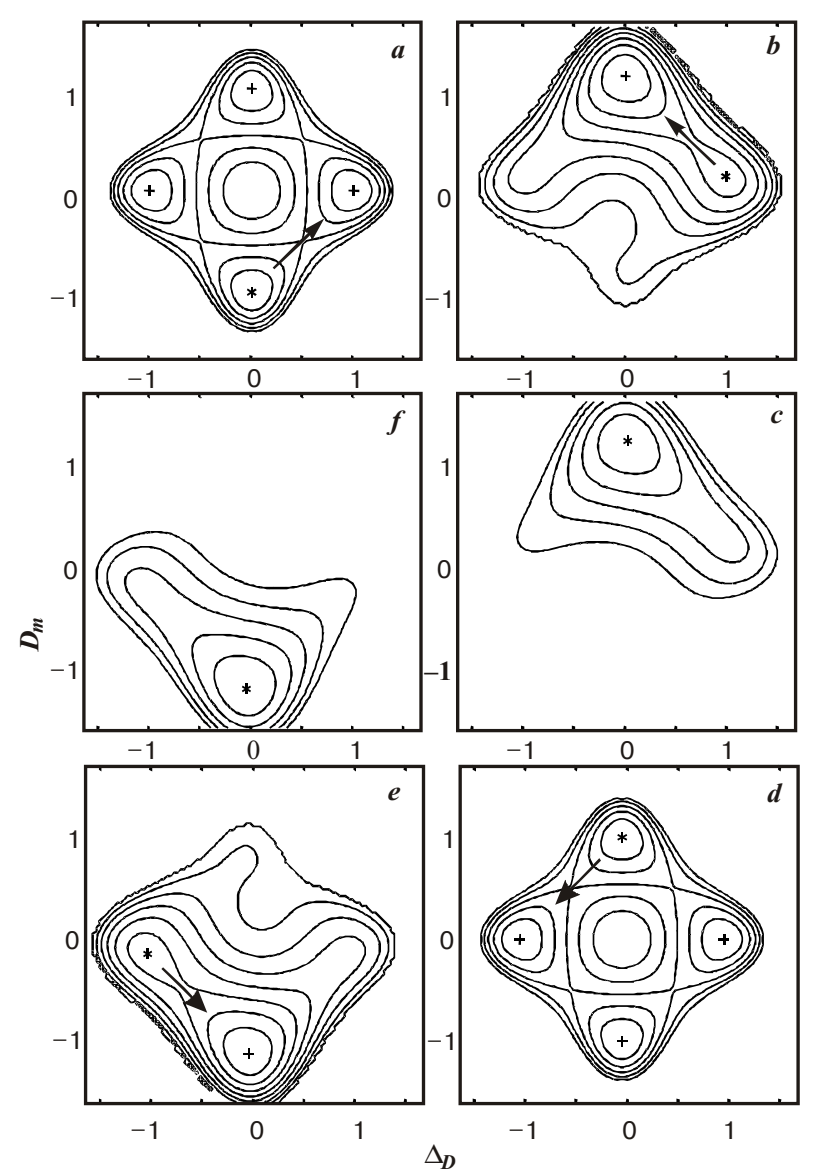

Fig. 2. The contour plots of negative values of the free energy (27) at $R^{2}=0.1$ and different values of $E_{m}=0,0.3,0.6,0,-0.3,-0.6$ (parts $a, b, c, d, e, f$, respectively). Crosses denote the positions of minima; asterisk is the position of the current minimum.

$\overline{\delta D^{2}} \approx 0$ (ordered state), and the second one with its zero value $\bar{D} \approx 0$ and $\overline{\delta D^{2}} \neq 0$ (disordered state). It is obvious that at zero external electric field all these four states have the same energy, and therefore the system can be in any of these states (see Fig. 1a,c). However, when one applies the external field $E_{0}$ above the definite threshold value, the states with induction direction opposite the field one vanish, and the system has to switch to the states with induction directed along the external field. In contrast to the pure ferroelectrics, where the switching takes place between two ordered states $\bar{D}= \pm 1$, the considered system from the ordered state $\bar{D}=1$ (Fig. 1c) switches first to the disordered one $\bar{D}=0$ (Fig. $1 d$ ) and vice versa (Fig. 1a,b). The plots in Fig. 1 correspond to the small amplitudes of the external field $E_{m}$, which can switch the system only between two adjacent minima denoted by the asterisk.

If the external field $E_{m}$ is increased, full switching between two ordered states $\bar{D}= \pm 1$ through disordered ones $\bar{D}=0$ is possible (see Fig. 2). In such case, the field $E_{m}$ can successively switch the system between four minima (e.g., from Fig. $2 a$ through Fig. $2 b$ to Fig. $2 c$, Fig. $2 d$ and then to Fig. $2 e, f)$. 


\section{A.N. Morozovska et al.: Partial polarization switching in ferroelectrics-semiconductors ...}

The contour plots of the free energy (27) at fixed $E_{m}$ and different $R$ values are depicted in Fig. 3. Again, free energy minima testify in favour of existence of the stable ordered and disordered states. The disordered state $\overline{\delta D^{2}} \neq 0$ is absent in pure ferroelectrics.

The cross-sections of the free energy (27) at $\overline{\delta D^{2}}=0$ (completely ordered states) and $\bar{D}=0$ (completely disordered states) at fixed $E_{m}$ and different $R$ values are depicted in Fig. 4. As it should be expected, the ordered state is more energetically preferable than the disordered one at $R^{2}<<1$ even in the infinitely small external field (see Figs $3 a, b$ and $4 a, b$ ). One can see that the probabilities of the system existence in ordered and disordered states become very close at $R^{2}>0.5$ (see Fig. $4 c$ ). Moreover, at $R \rightarrow 1$ ordered and disordered states become energetically indistinguishable (see Fig. $4 d$ ).

Our calculations proved that at $R>1$ disordered state with $\bar{D}=0, \overline{\delta D^{2}} \neq 0$ becomes the most energetically preferable even in the infinitely small external field. Actually, this means that the phase transition into the disordered state takes place at the critical value of fluctuations $\overline{\delta \rho_{s}^{2}}=\bar{\rho}_{s}^{2}$, i.e. the ferroelectrics sample with charged defects splits into the polar regions (domains or Cross regions [21]) with different induction orientations.

\section{Quasi-equilibrium dielectric hysteresis}

In this section, we demonstrate how the dielectric quasiequilibrium hysteresis loop $\bar{D}\left(E_{0}\right)$ changes its shape under the presence of charged defects. First of all, let us rewrite equation (26) in dimensionless variables:
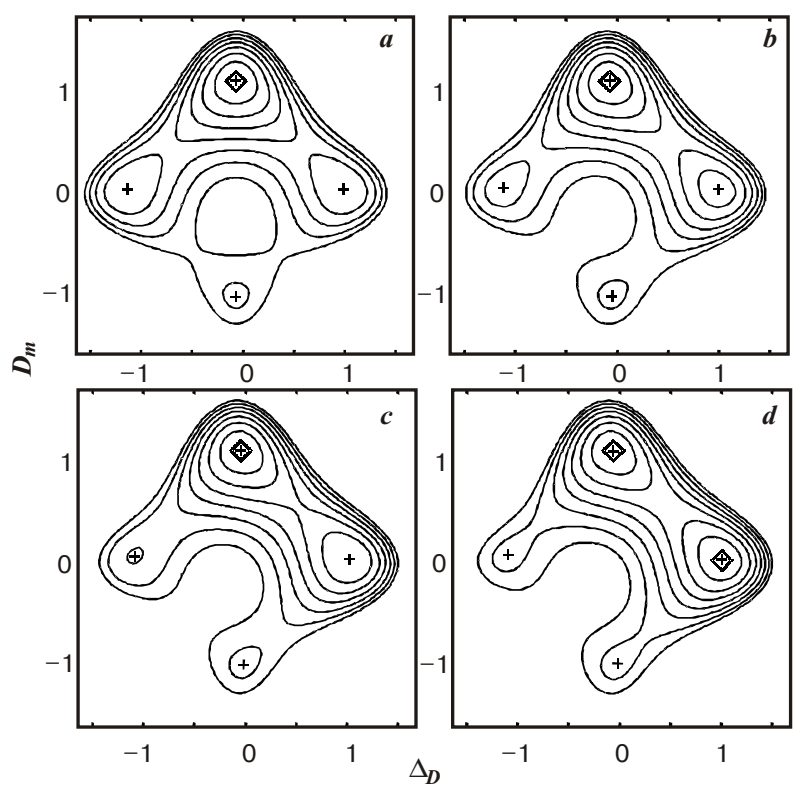

Fig. 3. The contour plots of negative values of the free energy (27) at $E_{m}=0.15$ and different values of $R^{2}=0,0.3,0.5,1$ (parts $a, b, c, d$, respectively). Crosses denote the positions of minima; marked crosses are the positions of the absolute minima.

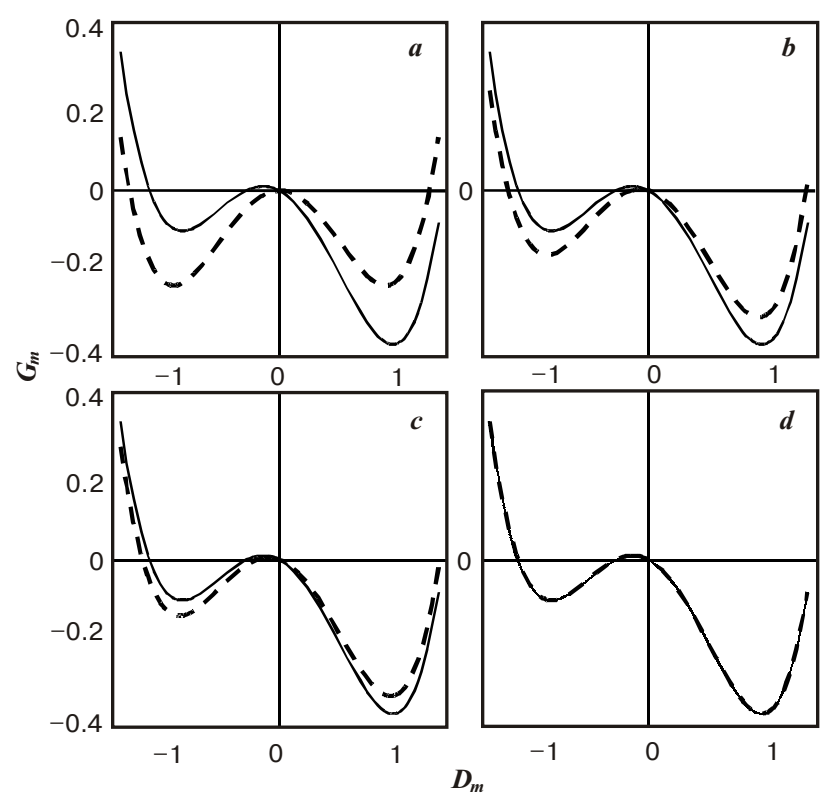

Fig. 4. The cross-sections of the free energy (27) at $\overline{\delta D^{2}}=0$ (solid curves) and $\bar{D}=0$ (dashed curves) for $E_{m}=0.15$ and different values of $R^{2}=0,0.3,0.5,1$ (parts $a, b, c, d$, respectively).

$\frac{d D_{m}}{d \tau}-\left(1-3 \Delta_{D}^{2}\right) D_{m}+D_{m}^{3}=E_{m}$,

$\frac{1}{2} \frac{d \Delta_{D}^{2}}{d \tau}-\left(1-3 D_{m}^{2}\right) \Delta_{D}^{2}+\Delta_{D}^{4}=-E_{m} K_{D \rho}$,

$\frac{d K_{D \rho}}{d \tau}-\left(1-3 D_{m}^{2}-\Delta_{D}^{2}\right) K_{D \rho}=-E_{m} R^{2}$.

Here $\alpha<0, D_{m}=\bar{D} / D_{S}, D_{S}=\sqrt{-\alpha / \beta}, E_{m}=E_{0} /\left(-\alpha D_{S}\right)$, $\Delta_{D}=\sqrt{\overline{\delta D^{2}}} / D_{S}, \quad K_{D \rho}=\overline{\delta D \delta \rho_{S}} / D_{S}^{2}, \quad \tau=t /(-\alpha \Gamma)$, $R^{2}=\overline{\delta \rho_{s}^{2}} / \bar{\rho}_{s}^{2}$. The dependence of the dimensionless induction $D_{m}$ over the external field $E_{m}$ is represented in Figs 5-9 for the case of harmonic modulation of the external field $E_{m}=E_{m A} \sin (w t)$. Hereinafter, we use the dimensionless frequency $w=-\alpha \Gamma \cdot \omega$.

The quasi-equilibrium hysteresis loops were obtained at the low frequency of external field (see Figs 5-6). It is seen from Figure 5 that a constriction on hysteresis loops appears for the nonzero $R$ values, and the loop area increases with the increase of $R$ parameter. The so-called "footprint" loops were observed in PLZT ceramics [10]. But for a given external field amplitude there is a critical value of $R$ parameter. For the $R$ value above critical one instead of "full" loops, we obtained only minor loops (see Fig. 6) or their absence (see Fig. $5 d$ ) depending on the ordered $\left(D_{m}(\tau=0)=1\right)$ or disordered $\left(D_{m}(\tau=0)=0\right)$ initial state of the sample. The similar minor loops were observed in PZT [1] and SBN: Ce single crystals [2].

On the other hand, there is a critical value of the external electric field amplitude for the given value of $R$ parameter. One can also see minor loops only for the amplitude of electrical field smaller than the critical one 


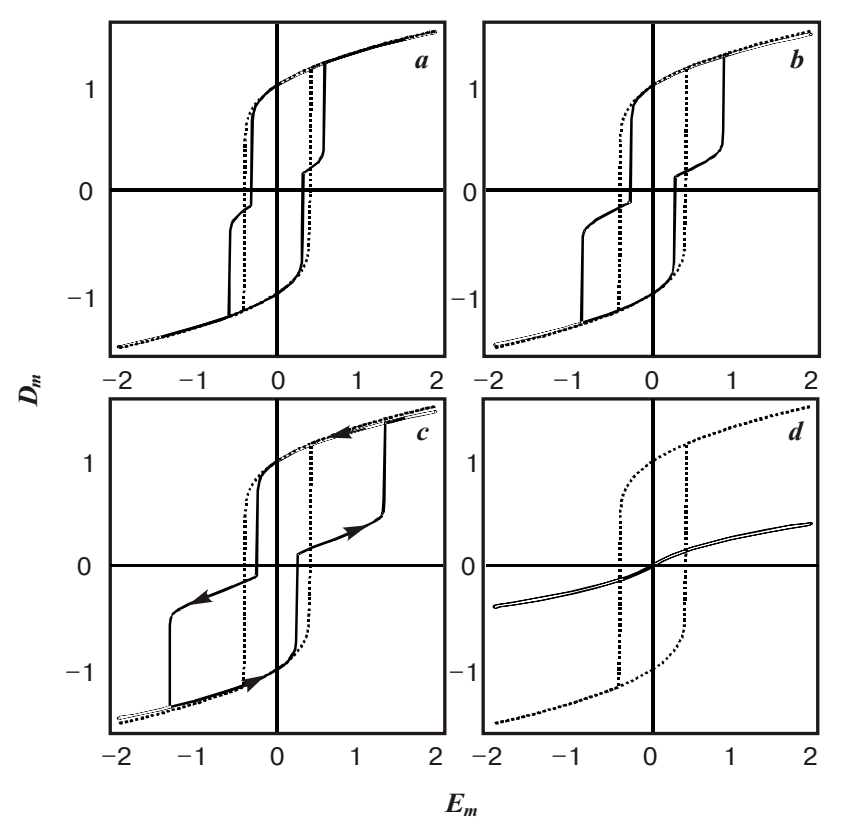

Fig. 5. The dependence of dimensionless induction on dimensionless field (hysteresis loops) for frequency $w=0.001$, external field amplitude $E_{m A}=2$ and different values of $R^{2}=0.1,0.3,0.5$, 1 (parts $a, b, c, d$, respectively). Initial conditions for the different solid curves are $\Delta_{D}^{2}(\tau=0)=0, K_{D \rho}(\tau=0)=0, D_{m}(t=0)=1$ (parts $a, b, c)$ and $D_{m}(\tau=0)=0$ (part $d$ ). The dotted curve corresponds to the "classic" loop in the pure ferroelectric $(R=0)$ at the same frequency value.

(compare Fig. 5 with Fig. 6). Even for the field amplitude below the thermodynamic coercive field $\left(E_{m C}=2 / 3 \sqrt{3} \approx 0.385\right)$, there is partial switching, when the hysteresis loop is absent in "pure" ferroelectrics (compare dotted and solid curves). It should be noted that for $R=1$ one can observe only minor loops (see Fig. $6 d$ ) for the finite value of the external electric field.

Note that two minor loops in Fig. 6a-d are obtained with several cycles of external field and switching between two loops is caused by the fluctuations. Upper minor loops represent solutions with the positive initial values of the induction, while the lower ones correspond to its negative initial values.

The hysteresis loops represented in Figs 7 and 8 are obtained with the same parameters as in Fig. 5, but with the higher frequency values. It is seen that Fig. 7 is qualitatively similar to the Fig. 5: there are constrictions on hysteresis loops for $R^{2} \leq 0.5$, but for $R=1$ only minor loop exists and paraelectric-like dependence $D(E)$ (see Fig. 5d) cannot be achieved. With the frequency increase, the constrictions are smeared and loops tend to those of pure ferroelectrics (see dotted lines in Fig. 8). Also under the frequency increase, minor loops arise for the smaller values of $R$ parameter at the same external field amplitude (compare Figs $7 c$ and $8 c$ ).

Switching between ordered and disordered states in ferroelectrics with charged defects causes both the minor hysteresis loops for the smaller external field and the con-
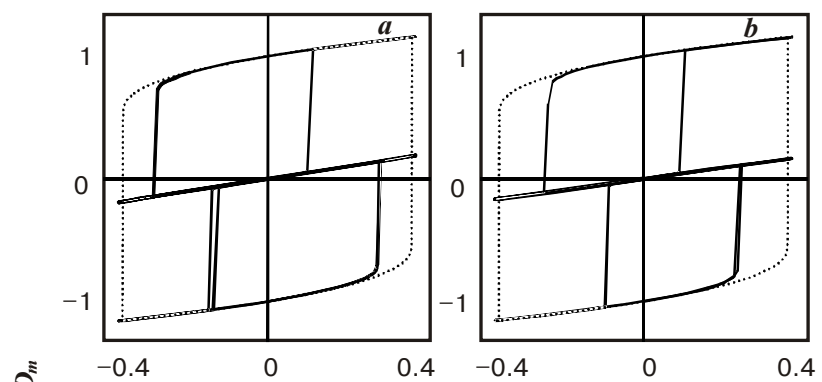

2

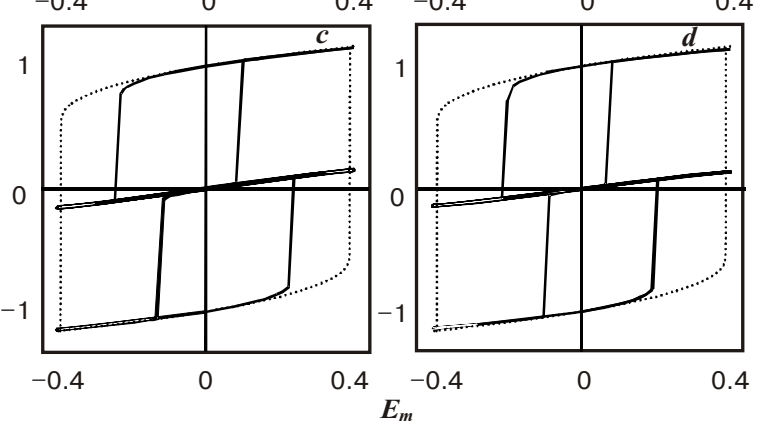

Fig. 6. Hysteresis loops for the frequency $w=0.001$, external field amplitude $E_{m A}=0.4$ and different values of $R^{2}=0.1,0.3$, $0.5,1$ (parts $a, b, c, d$, respectively). Initial conditions are $\Delta_{D}^{2}(\tau=0)=0, K_{D \rho}(\tau=0)=0, D_{m}(\tau=0)=-1$. The dotted curve corresponds to the "classic" loop in the pure ferroelectric $(R=0)$ at the same frequency value.

striction on loops for the larger external field amplitude. These phenomena can be easily explained on the basis of simple pictures of the free energy map evolution for different values of external field (see comments to Figs 1, 2). Therefore, our theory predicts footprint and minor polarization hysteresis loops in ferroelectric materials with charged impurities and relatively high improper conductivity (see e.g., $[1-3,8,10])$. It should be noted that the origin of the constricted or double loops in aged ferroelectric ceramics $\mathrm{BaTiO}_{3}$ and $(\mathrm{Pb}, \mathrm{Ca}) \mathrm{TiO}_{3}$ without charged impurities is caused by the mechanical clamping of spontaneous polarization switching [22], and so it lies outside of our theoretical consideration.

\section{The experimental results}

Let us compare the theoretically calculated dielectric response with our experimental results obtained for thick PZT films on Si substrate (see Scheme 3). The studied PZT-films with $\mathrm{Zr}$ /Ti ratio 54/46 are near to the morphotropic phase boundary, which corresponds to best performances for bulk PZT ceramics. Investigated Pt-PZT$\mathrm{Pt} / \mathrm{Ti}^{-\mathrm{SiO}_{2}} / \mathrm{Si}$ structures with oriented PZT layer were manufactured by RF magnetron sputtering in the system and under conditions described previously [25]. The sputtering target obtained by uniaxial cold pressing includes the mixture of $\mathrm{PbO}, \mathrm{TiO}_{2}$ and $\mathrm{ZrO}_{2}$ in a stochiometric 


\section{A.N. Morozovska et al.: Partial polarization switching in ferroelectrics-semiconductors ...}
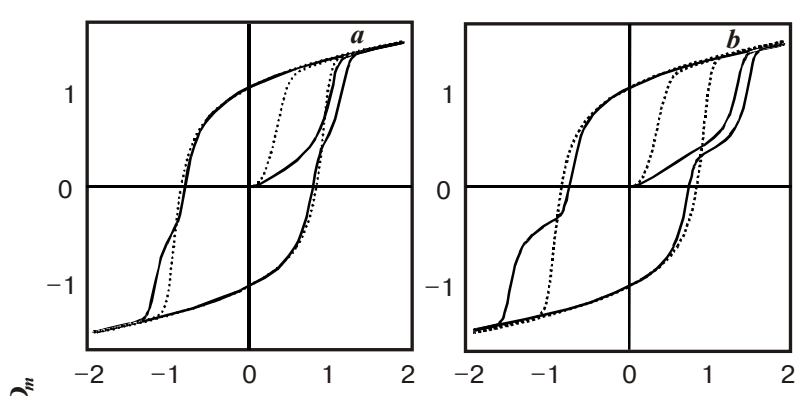

$\theta^{\Sigma}$

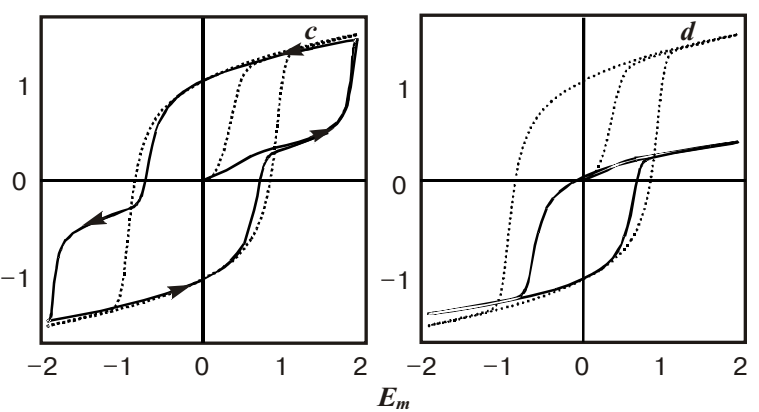

Fig. 7. Hysteresis loops for the frequency $w=0.1$, external field amplitude $E_{m A}=2$ and different values of $R^{2}=0.1,0.3,0.5,1$ (parts $a, b, c, d$, respectively). Initial conditions are $\Delta_{D}^{2}(\tau=0)=1$, $K_{D \rho}(\tau=0)=0, D_{m}(\tau=0)=0$. The dotted curves corresponds to the "classic" loops in the pure ferroelectric $(R=0)$ at the same frequency value.

composition. The structure includes the top $150 \mathrm{~nm} \mathrm{Pt}$ electrode, $1.9 \mu \mathrm{m}$ layer of (111)-oriented PZT, bottom $\mathrm{Pt} /$ Ti-bilayer (150 nm of Pt, $10 \mathrm{~nm}$ of Ti) deposited onto the oxidized (350 nm of $\mathrm{SiO}_{2}$ ) (100) $n$-type $\mathrm{Si} 350 \mu \mathrm{m}$ substrate.

For PZT - Si-substrate structure, it is necessary to design the bottom electrode, which possesses not only a stable and high enough electrical conductivity but also simultaneously prevents the interfacial reactions between electrode, PZT and $\mathrm{SiO}_{2}$ components in PZT-film and Si-substrate surroundings under rather high temperature. The layer of Ti plays an important role in limiting the diffusion of Ti in Pt/Ti intermediate bilayer through Ptlayer into the PZT-layer and directly into $\mathrm{SiO}_{2}$-layer, and also in correction of poor adhesion of Pt-layer. The

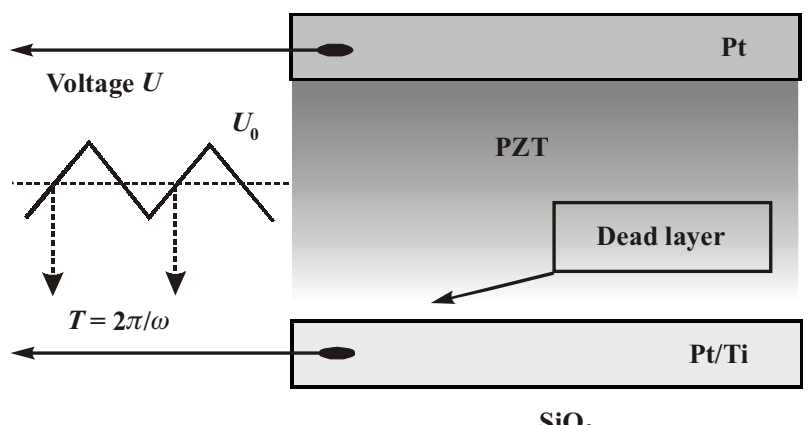

Scheme 3. The PZT film on Si substrate, $U$ is the applied voltage. SQO, 7(3), 2004
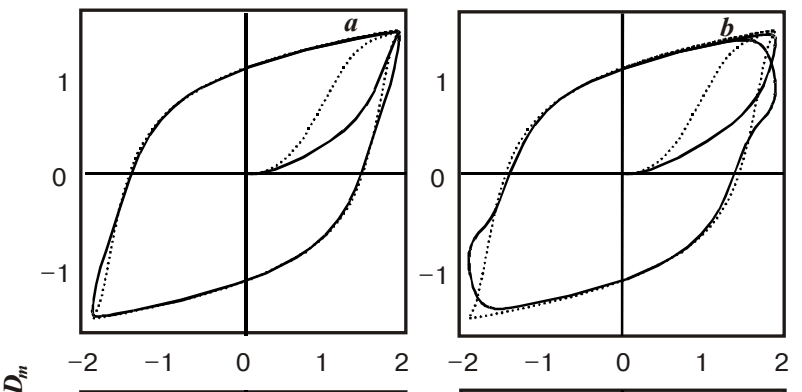

$\theta$

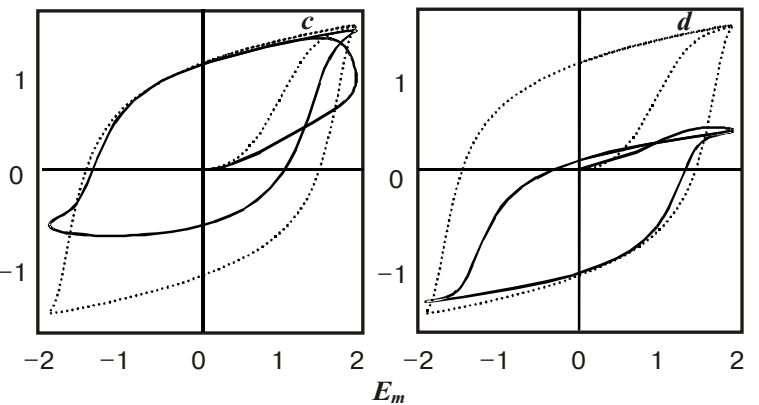

Fig. 8. Hysteresis loops for the frequency $w=0.5$, external field amplitude $E_{m A}=2$ and different values of $R^{2}=0.1,0.3,0.5,1$ (parts $a, b, c, d$, respectively). Initial conditions are $\Delta_{D}^{2}(\tau=0)=1$, $K_{D \rho}(\tau=0)=0, D_{m}(\tau=0)=0$. The dotted curves correspond to the "classic" loop in the pure ferroelectric $(R=0)$ at the same frequency value.

annealing treatment of the $\mathrm{Pt} / \mathrm{TiO}_{\mathrm{x}} / \mathrm{SiO}_{2} / \mathrm{Si}$-substrate structure just before of PZT deposition was performed for substrate stabilization and post-annealing treatment of PZT-film was performed for crystallizing the film in the polar perovskite phase. The top Pt-electrodes have $1 \mathrm{~mm}^{2}$ area.

The ferroelectric hysteresis loops for the PZT films were obtained using the conventional Sawyer-Tower circuit (see, e.g., [16]), and the bipolar triangular voltage $U$ (symmetrical saw-tooth) with the frequency about $1 \mathrm{KHz}$ was applied (see Scheme 3).

For the most of the films, the hysteresis loops had rather "slim" than "square" shape and were strongly asymmetric. The typical minor loop is represented in Fig. 9. Notice that mm-thick semiconductor films are thick enough to neglect the size-driven effects of dielectric properties changing [23], and partial switching phenomena are caused by other reasons. In our model, the high fluctuations of the charged defects concentration can lead to the partial switching of the induction. In the considered material, the source of this charged defects can be related to the numerical randomly distributed $\mathrm{Pb}$ vacancies (so $R \sim 1$ ) appeared during the high temperature annealing due to the high $\mathrm{PbO}$ volatility.

One can see from Fig. 9 that proposed model quantitatively well describes experimental data. Some discrepancy between the theory and experiment in the vicinity of the coercive field values can be related to the mobility of charged defects under the external field high values, which are treated as static in our model, as well as to the dead layer appearance [1]. 


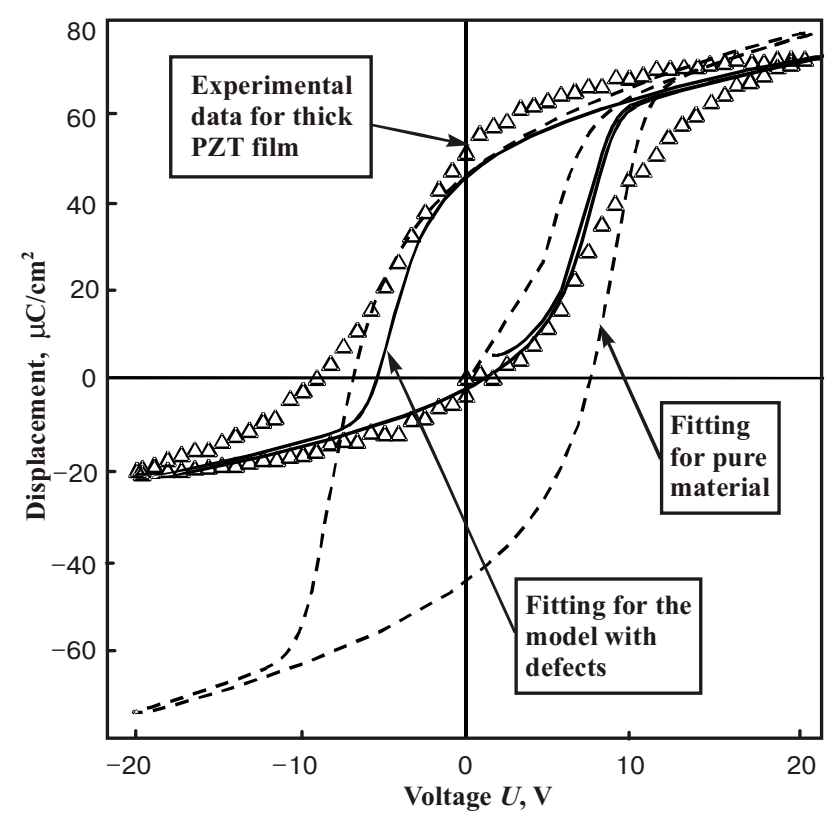

Fig. 9. Minor loop observed in a thick ferroelectric PZT film $(\ell=1.9 \mu \mathrm{m})$ for the triangular applied voltage. Triangles are experimental data measured at $U_{0}=20 \mathrm{~V}$, solid curve is our fitting for the material with charged defects $(R=0.95)$ at $w=0.35$, $D_{S}=40 \mu \mathrm{C} / \mathrm{cm}^{2}, U_{C}=4 V$. The dotted curve corresponds to the loop in pure ferroelectric $(R=0)$ at the same other parameters.

The dielectric permittivity hysteresis calculated by means of the data in Fig. 9 is shown in Fig. 10. The dielectric permittivity $\varepsilon=\ell \cdot d \bar{D} / d U$ was calculated numerically by the simple central difference without smoothing. It is seen from the figure that slight variation of the experimental data in Fig. 9 led to the anomalous oscillations of permittivity, which have no physical meaning.

The capacity of a $1.9 \mu \mathrm{m}$-thick ferroelectric PZT film for triangular applied voltage has been measured independently at the lower frequencies and higher applied voltage amplitudes. The typical capacity hysteresis is depicted in Fig. 11. It is seen that the dead layer influence increases under external voltage increasing. The dead layer has been taken into account as the effective series capacity $C_{d}$ (see Scheme 3 ), thus the resulting measured capacity $C=C_{F} \cdot C_{d} /\left(C_{F}+C_{d}\right)$ differs from the film capacity $C_{F}$.

We can conclude that coupled equations (24) describe the polarization switching and ferroelectric disordering caused by charged defects in the thick ferroelectric PZT films with $\mathrm{Pb}$ vacancies.

\section{Dicussion}

We have proposed the phenomenological description of polarization switching peculiarities in some ferroelectric semiconductor materials with charged defects. The comparison with our experimental results obtained for thick PZT films has been performed.

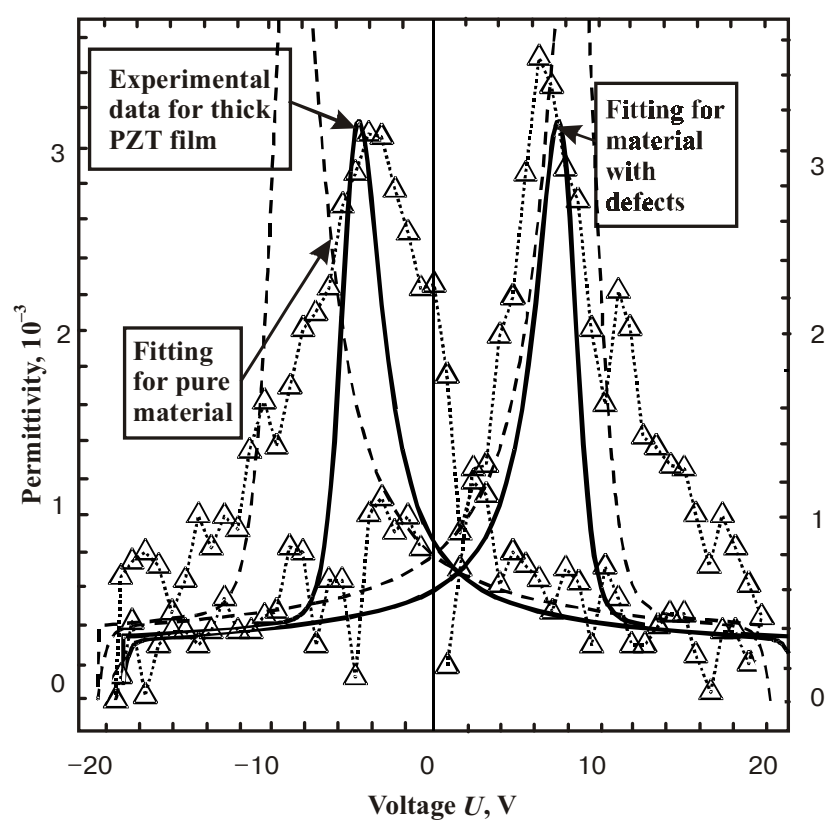

Fig. 10. The dielectric permittivity hysteresis of $1.9 \mu \mathrm{m}$-thick ferroelectric PZT film for triangular applied voltage. Triangles are experimental data calculated from the loop in Fig. 9, solid curve is our fitting for the material with charged defects $(R=0.95)$ at $U_{0}=20 \mathrm{~V}, w=0.35, D_{S}=40 \mu \mathrm{C} / \mathrm{cm}^{2}, U_{C}=4 \mathrm{~V}$. The dotted curve corresponds to the hysteresis in pure ferroelectric $(R=0)$ at the same other parameters.

It is shown that the impurity concentration fluctuations $\delta \rho_{s}(\mathbf{r})$ result in to the ferroelectric disordering of the considered system. The quantitative degree of this disorder is the parameter $\sqrt{\overline{\delta D^{2}}}$ characterizing the inhomogeneity of the induction distribution. The mean induction $\bar{D}$ is the order parameter. For the first time, the system of coupled equations (24) that determines the evolution of these parameters has been derived.

Solving the system of coupled equations one can get the information about system ordering as a whole, without defining concrete space distribution of the appeared inhomogeneities, domain walls characteristics, correlation radius of Cross regions or sizes of originated microdomains. In order to obtain this kind of information, one has to solve the system of equations (13)-(16) with the specified distribution of impurity concentration fluctuations $\delta \rho_{s}(\mathbf{r})$, but the consideration of this problem was not the purpose of the present paper.

We would like to underline that in contrast to the system (13)-(16) the averaged system of the coupled equations (24) does not contain any information about induction gradient across the sample. This happened rather due to the local compensation of the strong inhomogeneous electric field in the vicinity of charged defects by the movable charge carriers, then due to enough sample thickness in order to neglect the size effects and the depolarization field influence [23], [24]. We also suppose that inhomogeneous mechanical stresses arisen near defects are rather small or compensated by the sample treatment. 


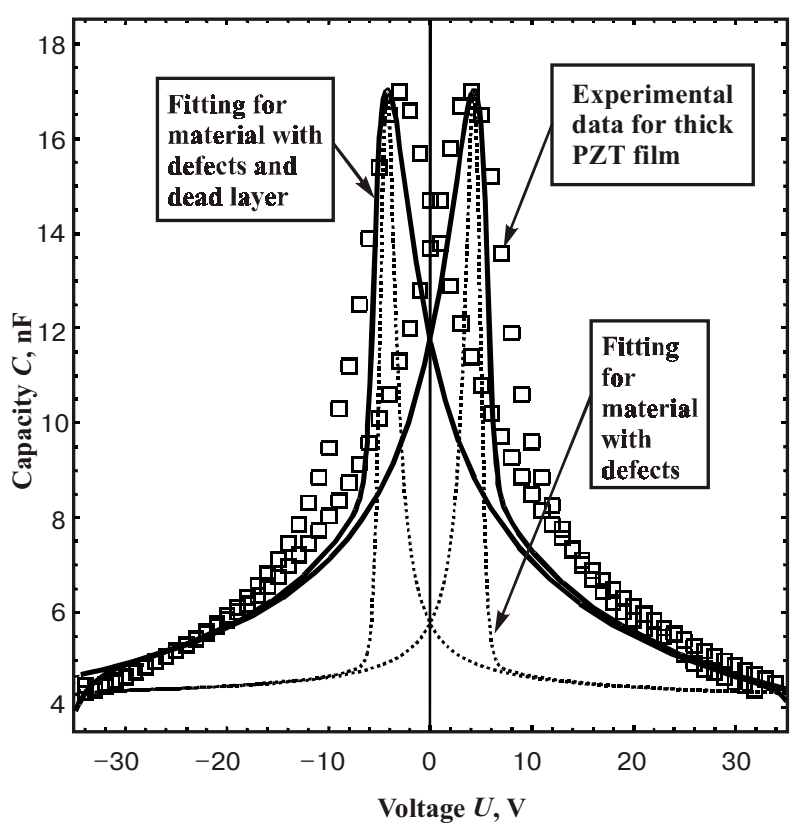

Fig. 11. The capacity hysteresis of a $1.9 \mu \mathrm{m}$-thick ferroelectric PZT film for triangular applied voltage. Squares are experimental data measured at $U_{0}=36 \mathrm{~V}$, solid curve is our fitting for the material with charged defects $(R=0.95)$ at $w=0.1, D_{S}=40 \mu \mathrm{C} / \mathrm{cm}^{2}$, $U_{C}=4 \mathrm{~V}$. The dotted curve is our fitting for the material with charged defects and dead layer with capacity $C_{d}=0.16 \mathrm{nF}$ at the same other parameters.

In accord to our theory, random inhomogeneities in the defect distribution throughout the sample lead to the stabilization of the disordered state $\left(\sqrt{\overline{\delta D^{2}}}>\bar{D}\right)$. For sufficiently small external field amplitudes, this state reveals itself as switching from the ordered state to the disordered one (the so-called minor loop, see e.g. [1]). When the external field increases this minor loop transforms into the loop with constriction or footprint-type hysteresis loop [9]. In this case, the constriction corresponds to the switching from the ordered state to disordered one and then again to the ordered state with the opposite direction of the induction. Ferroelectric hysteresis loops with the constrictions or footprint loops are observable in some ferroelectric materials. For example, footprint loops exist in the plumbum zirconate-titanate ceramics doped with La [10], namely in at $x=0.35, y=0.08,0.084$ and $x=0.3, y=0.076,0.079$, which is regarded as relaxor material. In this material, La ions have excess charge and can be regarded as charged defects. Notice that our theory predicts transformation from footprint to the minor loop with the external field frequency increase.

The transformation from full loop to the minor one was observed in SBN single crystals doped with cerium under applied field frequency increasing (see Fig. 6 in [2]). It is clear that our theory [20] describes qualitatively minor loops observed in SBN:Ce [2], $\mathrm{Pb}(\mathrm{Zr}, \mathrm{Ti}) \mathrm{O}_{3^{-}}$
$\mathrm{Pb}(\mathrm{Sb}, \mathrm{Mn}) \mathrm{O}_{3}$ ceramics [3], PZT thin films [8], thick PZT films with $\mathrm{Pb}$ vacancies [25] and TGS [5], but not the aging process seen as the loop degradation. This may be related to the fact that neither finite domain wall thickness $\ell_{c}$, nor possible evolution of the charge fluctuations $\delta \rho_{s}$ caused by the relaxation/origin of internal stresses around defects was taken into account in our model. These problems as well as the calculation of the system dielectric response are in progress now.

We can conclude that coupled equations (24) qualitatively describe the polarization switching and ferroelectric disordering caused by charged defects in bulk ferroelectric-semiconductors.

\section{Acknowledgments}

The authors are greatly indebted to Profs. S.L. Bravina and N.V. Morozovsky for frutfull discussions of the model and useful remarks to the manuscript.

\section{Appendix A}

Let us express the field variation $\delta E_{z}$ can be via $\delta D$ and $\delta \rho_{s}$. In accordance with (21b) and (20), one obtains that $\delta n \approx(1 / 4 \pi) \partial \delta D / \partial z-\delta \rho_{s}$. Having substituted this expression into (21a), one obtains (23) using the inequality (22):

$$
\begin{aligned}
& \delta E_{z} \approx\left(E_{0}(t)-\frac{\kappa}{\mu} \frac{\partial}{\partial z}\right)\left(\frac{1}{4 \pi \bar{\rho}_{s}} \frac{\partial}{\partial z} \delta D-\frac{\delta \rho_{s}}{\bar{\rho}_{s}}\right) \approx \\
& \approx \frac{\kappa}{\mu} \frac{\partial}{\partial z}\left(\frac{\delta \rho_{s}}{\bar{\rho}_{s}}\right)-E_{0}(t) \frac{\delta \rho_{s}}{\bar{\rho}_{s}} .
\end{aligned}
$$

The equations for $\overline{\delta D^{2}}$ and $\overline{\delta D \delta \rho_{s}}$ obtained directly from (16) have the form:

$$
\begin{aligned}
& \frac{\Gamma}{2} \frac{\partial}{\partial t} \overline{\delta D^{2}}+\left(\alpha+3 \beta \bar{D}^{2}(t) \overline{\delta D^{2}}+\right. \\
& +\beta \overline{\delta D^{4}}=\gamma \delta D \frac{\partial^{2} \delta D}{\partial \mathbf{r}^{2}}+\overline{\delta D \delta E_{z}}, \\
& \Gamma \frac{\partial}{\partial t} \overline{\delta D \delta \rho_{s}}+\left(\alpha+3 \beta \bar{D}^{2}(t) \overline{\delta D \delta \rho_{s}}+\right. \\
& +\beta \overline{\delta D^{3} \delta \rho_{s}}=\gamma \delta \rho_{s} \frac{\partial^{2} \delta D}{\partial \mathbf{r}^{2}}+\overline{\delta \rho_{s} \delta E_{z}}
\end{aligned}
$$

One can derive from (A.1) the following approximations for the correlations:

$$
\begin{aligned}
& {\overline{\delta D \delta E_{z}}}=-E_{0}(t) \frac{\overline{\left(\delta \rho_{s} \delta D\right)}}{\bar{\rho}_{s}}+\frac{\kappa}{\mu\left(\delta D \frac{\partial}{\partial z} \frac{\delta \rho_{s}}{\bar{\rho}_{s}}\right)} \approx \\
& \approx-E_{0}(t) \frac{\overline{\left(\delta \rho_{s} \delta D\right)}}{\bar{\rho}_{s}}
\end{aligned}
$$


In (A.4) the term $\frac{\kappa}{\mu\left(\delta D \frac{\partial}{\partial z} \frac{\delta \rho_{s}}{\bar{\rho}_{s}}\right)} \sim \frac{\kappa}{\mu \bar{\rho}_{s} d} \overline{\left(\delta \rho_{s} \delta D\right)}=$ $=\frac{4 \pi \varepsilon_{\perp} R_{D}^{2}}{d} \overline{\left(\delta \rho_{s} \delta D\right)}$ can be neglected under the assumption that the screening of defects is rather strong to satisfy the inequality $R_{D}^{2} / d^{2}<<E_{0} /\left(4 \pi \varepsilon_{\perp} d \bar{\rho}_{s}\right)$ (see (20)). For a thick sample with equivalent boundaries $z= \pm \ell$ we obtain from (A.1) that

$$
{\overline{\delta \rho_{s} \delta E}}_{z}=-E_{0}(t) \frac{\overline{\delta \rho_{s}^{2}}}{\bar{\rho}_{s}}+\frac{\kappa}{2 \mu} \overline{\left(\frac{\partial}{\partial z} \frac{\delta \rho_{s}^{2}}{\bar{\rho}_{s}}\right)} \equiv-E_{0}(t) \frac{\overline{\delta \rho_{s}^{2}}}{\bar{\rho}_{s}} .
$$

Taking into account (20) one obtains that

$$
\begin{aligned}
& \gamma \overline{\delta D \frac{\partial^{2} \delta D}{\partial \mathbf{r}^{2}}}=-\gamma \overline{\left(\frac{\partial \delta D}{\partial \mathbf{r}}\right)^{2}} \cong-\frac{\gamma}{d^{2}} \overline{\delta D^{2}}, \\
& \left|\frac{\gamma}{d^{2}} \overline{\delta D^{2}}\right|_{<<|\alpha|} \overline{\delta D^{2}},
\end{aligned}
$$

$$
\begin{gathered}
\gamma \delta \rho_{s} \frac{\partial^{2} \delta D}{\partial \mathbf{r}^{2}} \sim-\frac{\gamma}{d^{2}} \overline{\delta \rho_{s} \delta D}, \\
\left|\frac{\gamma}{d^{2}} \overline{\delta \rho_{s} \delta D}\right|<<\left|\alpha \overline{\delta \rho_{s} \delta D}\right|,
\end{gathered}
$$

and so gradient terms in (A.2)-(A.3) can be either neglected at $\gamma / d^{2}<<\alpha$ or the coefficient a can be renormalized as $\alpha \rightarrow \alpha_{R}=\left(\alpha+\gamma / d^{2}\right)$. One obtains also from (9)-(10) that

$$
\overline{\delta D^{4}} \approx\left(\overline{\delta D^{2}}\right)^{2}, \overline{\delta D^{3} \delta \rho_{s}} \approx \overline{\delta D^{2}} \overline{\delta D \delta \rho_{s}} .
$$

Using (A.3)-(A.6) we obtain the equations (26b) and (26c) from the equations (A.1) and (A.2) if only $\gamma / d^{2}<<\alpha$ (see (20)).

\section{References}

1. J.C. Burfoot, G.W. Taylor, Polar dielectrics and their applications, chapter 3, p. 41, The Macmillan Press, London (1979).

2. T.Granzow, U.Dorfler, Th.Woike, M.Wohlecke, R.Pankrath, M. Imlau, W. Kleemann, Influence of pinning effects on the ferroelectric hysteresis in cerium-doped $\mathrm{Sr}_{0.61} \mathrm{Ba}_{0.39} \mathrm{Nb}_{2} \mathrm{O}_{6} / /$ Phys. Rev. B 63(17), p. 174101(7) (2001).

3. Y. Gao, K. Uchino, D. Viehland, Effects of rare earth metal substituents on the piezoelectric and polarization properties of $\mathrm{Pb}(\mathrm{Zr}, \mathrm{Ti}) \mathrm{O}_{3}-\mathrm{Pb}(\mathrm{Sb}, \mathrm{Mn}) \mathrm{O}_{3}$ ceramics // J. Appl. Phys. 92(4), pp.2094-2099 (2002).
4. E. Tokumitsu, N. Tanisake, H. Ishiwara, Partial switching kinetics of ferroelectric $\mathrm{PbZr}_{\mathrm{x}} \mathrm{Ti}_{1-\mathrm{x}} \mathrm{O}_{3}$ thin films prepared by sol-gel technique // Jpn. J. Appl. Phys. 33(9B), pp. 52015206 (1994).

5. P. Wurfel and I.P. Batra, Depolarization field induced instability in thin ferroelectric films - experiment and theory // Phys. Rev. B, 8(11), pp. 5126-5133 (1973).

6. I.P. Batra, P. Wurfel and B.D. Silverman, Phase transition, stability and depolarization field in ferroelectric thin films // Phys. Rev. B, 8(7), pp. 3257-3265 (1973).

7. Y. Watanabe, A. Masuda, Ferroelectric self-field effect: implications for size effect and memory device // Integrated Ferroelectrics, 27, pp. 51-60 (1999).

8. T. Tamura, K. Matsuura, H. Ashida, K. Konda, S. Otani, Hysteresis variations of $(\mathrm{Pb}, \mathrm{La})(\mathrm{Zr}, \mathrm{Ti}) \mathrm{O}_{3}$ capacitors baked in a hydrogen atmosphere // Appl. Phys. Lett. 74(22), pp. 3395-397 (1999).

9. J.C. Burfoot, Pyroelectric and ferroelectric thin film devices, chapter 12 , p.731 in "Active and passive thin film devices", pp. 697-741, London (1978)

10. Q.Y. Jiang, E.C. Subbarao and L.E. Cross, Effect of compositional and temperature on electric fatigue of La-doped lead zirconate titanate ceramics // J. Appl. Phys. 75(11), pp. 7433-7443 (1994)

11. V. Westphal, W. Kleemann and M.D. Glinchuk. // Phys. Rev. Lett., 68 p. 847 (1992).

12. H. Haken, Synergetics, chapter 12, Springer-Verlag, Berlin (1978).

13. V.A. Zhirnov, Contribution to the theory of domain walls in ferroelectrics // Sov. Phys. JETP, 35(5), pp. 822-827 (1959).

14. N.A. Pertsev, A.G. Zembilgotov, A.K. Tagantsev, Effect of Mechanical Boundary Conditions on Phase Diagrams of Epitaxial Ferroelectirc Thin Films // Phys. Rev. Lett., 80, pp. 1988-1991 (1998).

15. V.M. Fridkin, Ferroelectrics semiconductors, chap.3, Consultant Bureau, New-York and London (1980).

16. M.E. Lines and A.M. Glass, Principles and Applications of Ferroelectrics and Related Phenomena, Oxford University Press, Oxford (1978)

17. G.M. Guro, I.I. Ivanchik, N.F. Kovtonuk, Semiconducting properties of barium titanate // Fiz. Tverd. Tela, 10(1), pp. 134143 (1968) [Sov. Phys.-Solid State, 10(1), pp. 100-109 (1968)].

18. R.Landauer, Electrostatic consideration in $\mathrm{BaTiO}_{3}$ domain formation during polarization reversal // J. Appl. Phys., 28(2), pp. 227-234 (1957).

19. R.C.Miller and G.Weinreich, Mechanism for the sidewise motion of $180^{\circ}$ domain walls in barium titanate // Phys. Rev., 117(6), pp. 1460-1466 (1960).

20. Morozovska A.N., Eliseev E.A., Phenomenological Description of Disordering in Ferroelectric Materials Caused by Charged Defects// http://arXiv.org, cond-mat/0404073 (2004)

21. L.E. Cross, Relaxor ferroelectrics // Ferroelectrics, 76, pp. 241-245 (1987)

22. B. Jaffe, W.R. Cook and H. Jaffe, Piezoelectric Ceramics, chapter 3, p. 49, Academic Press, London and New York (1971).

23. M.D. Glinchuk, E.A. Eliseev, V.A. Stephanovich, The depolarization field effect on the thin ferroelectric films properties // Physica B, 332, pp. 356-370 (2002).

24. M.D. Glinchuk, E.A. Eliseev, V.A. Stephanovich, R. Fahri, Ferroelectric thin films properties - Depolarization field and renormalization of a "bulk" free energy coefficients // $J$. Appl. Phys., 93(2), pp. 1150-1159 (2003).

25. T. Haccart, E. Cattan, D. Remiens, Dielectric, ferroelectric and piezoelectric properties of sputtered PZT thin films on Si substrates: influence of film thickness and orientation // Semiconductor Physics, Quantum Electronics \& Optoelectronics, 5 (1), pp 78-88 (2002) 